\title{
Valutazione antropometrica e bioimpedenziometrica del bambino affetto da insufficienza renale cronica (IRC)
}

\author{
P. Sacco, F. Perfumo, P. Delucchi, R. Gusmano \\ Divisione di Nefrologia, Dialisi e Trapianto, Istituto Scientifico G. Gaslini - Genova
}

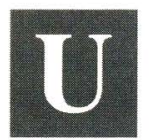

no stato di malnutrizione può essere osservato con particolare frequenza nel corso di malattie renali e in particolare in presenza di insufficienza renale $(8,26,32,41,44,47)$. Numerose sono le cause che possono determinarne l'insorgenza e tra queste le più importanti sono rappresentate da:

- ridotto apporto alimentare conseguente ad anoressia, disgeusia, restrizioni dietetiche eccessive, cibi poco palatabili, depressione psicologica, farmaci, malattie sovraimposte;

- alterazioni del metabolismo degli aminoacidi e di altri nutrienti;

- anomalie endocrine (insulino-resistenza, iperglucagonemia, iperparatiroidismo);

- tossine uremiche;

- stati di ipercatabolismo.

È quindi molto importante saper scoprire e caratterizzare uno stato di malnutrizione del bambino uremico al fine di poter istituire tempestivamente un adeguato supporto nutrizionale. A tale scopo i principali metodi di valutazione proposti per il soggetto con insufficienza renale sono rappresentati dalla registrazione degli apporti alimentari, dalla valutazione di parametri ematochimici, antropometrici e indicatori della composizione corporea. Dobbiamo peraltro ricordare che nessuna singola misurazione attualmente utilizzata nella routine clinica è in grado di riflettere accuratamente lo stato nutrizionale dell'organismo, ma sono necessari diversi tipi di valutazioni per ottenere informazioni sufficientemente indicative. Scopo del nostro studio è stato quello di verificare il comportamento dello stato nutrizionale in un gruppo di bambini affetti da insufficienza renale cronica (IRC) seguiti longitudinalmente per 3 anni.

\section{Materiale e metodi}

Sono stati valutati 25 bambini (14 maschi e 11 femmine) affetti da IRC di età compresa tra 1 e 16 anni (età media all'inizio dello studio $8.81 \pm 3.74$ anni) e con un filtrato glomerulare (GFR), calcolato secondo la formula di Schwartz, inferiore a $50 \mathrm{ml} / \mathrm{min} / \mathrm{m}^{2} 1.73$ (media $32.48 \pm$ 13.68) seguiti per un periodo di 36 mesi. La malattia renale primitiva era rappresentata da: nefropatia da reflusso (13 pazienti), displasia renale (4 pz.), malattia policistica (3 pz.), sindrome emoliticouremica (2 pz.), uropatia ostruttiva (2 pz.) e nefronoftisi (1 pz.). Ogni tre-sei mesi venivano valutati, oltre alla funzione renale, parametri antropometrici, bioimpedenziometrici ed ematochimici. I parametri antropometrici rilevati sono stati la statura, il peso, la plica tricipitale
(TSF), la plica sottoscapolare (SSF) e la circonferenza del braccio (MAC). Da questi elementi sono stati successivamente derivati:

- Body Mass Index (BMI): $\frac{\text { peso }(\mathrm{kg})}{\text { altezza }(\mathrm{m})^{2}}$

- Circonferenza muscolare del braccio (MAMC): MAC - $\pi$ TSF

- Area trasversale del muscolo dell'arto superiore (AMA): MAMC $^{2}$

$4 \pi$

- Area trasversale del grasso dell'arto superiore (AFA): $\mathrm{MAC}^{2}-\mathrm{AMA}$

$$
4 \pi
$$

Statura e peso sono stati valutati con il metodo dello Z-score (standard deviation score, SDS) che viene calcolato con la seguente formula

SDS: valore osservato - valore al $50^{\circ}$ percentile per età e sesso

$\mathrm{DS}$ al $50^{\circ}$ percentile per età e sesso

Per quanto riguarda i parametri impedenziometrici, la resistenza $(\mathrm{Rx})$ e la reattan$\mathrm{za}(\mathrm{Xc})$ sono state valutate con un analizzatore BIA 101 (Rjl System, Detroit), 
utilizzando una corrente elettrica alternata di $800 \mathrm{~mA}$ e $50 \mathrm{KHz}$ che attraversa l'organismo da una coppia di elettrodi ad un'altra coppia di elettrodi tra la mano e il piede omolaterale.

Ogni misurazione è stata effettuata in posizione supina con gli arti superiori rilassati posti lungo il tronco, senza che vi fosse contatto con esso, e le gambe leggermente divaricate. Attraverso l'utilizzo di un software impiegante equazioni predittive dalla Rx e dalla Xc sono stati calcolati:

- Massa Magra (FFM)

- Massa Cellulare (BCM)

- Acqua Corporea Totale (TBW)

- Acqua Extracellulare (ECW).

I parametri ematochimici valutati sono stati: bicarbonatemia $\left(\mathrm{HCO}_{3}\right)$, colesterolemia, trigliceridemia, proteinemia e albuminemia. In tutti i pazienti sono stati rilevati, ogni sei mesi, gli apporti alimentari mediante registrazione dietetica effettuata per almeno tre giorni consecutivi. Sono stati così valutati l'apporto di proteine, espresso sia in grammi $/ \mathrm{kg}$ peso corporeo/die sia in percentuale delle RDA (Recommended Dietary Allowances), e l'apporto calorico, espresso in Kcalorie/kg peso corporeo/die e in percentuale delle RDA. Tutti i valori percentuali sono stati calcolati in base all'età cronologica. L'analisi statistica è stata eseguita applicando il t di Student per dati appaiati.

\section{Risultati}

Nella Figura 1 è riportato l'andamento della funzione renale espresso come clearance della creatinina. Gli apporti dietetici non sono variati in modo significativo nel corso dell'osservazione. L'apporto proteico presentava limiti estesi, compresi tra 64 e $180 \%$ delle RDA, con valori medi ai diversi tempi tra 97 e $107 \%$; l'apporto calorico risultava inferiore alla quota prescritta con, anche in questo caso, ampie differenze. I valori medi erano compresi tra 78.9 e $90.8 \%$ delle RDA. I dati riguardanti statura e peso sono presentati nella Figura 2. Il ritardo di crescita si è mantenuto attorno a valori di -1.2 DS, il peso è risultato essere costantemente attorno a valori di -0.9/-1.1 DS. Per entrambi i parametri non vi erano differenze significative tra $\mathrm{i}$ diversi periodi di osservazione ad eccezione della statura rilevata al tempo 12 mesi.

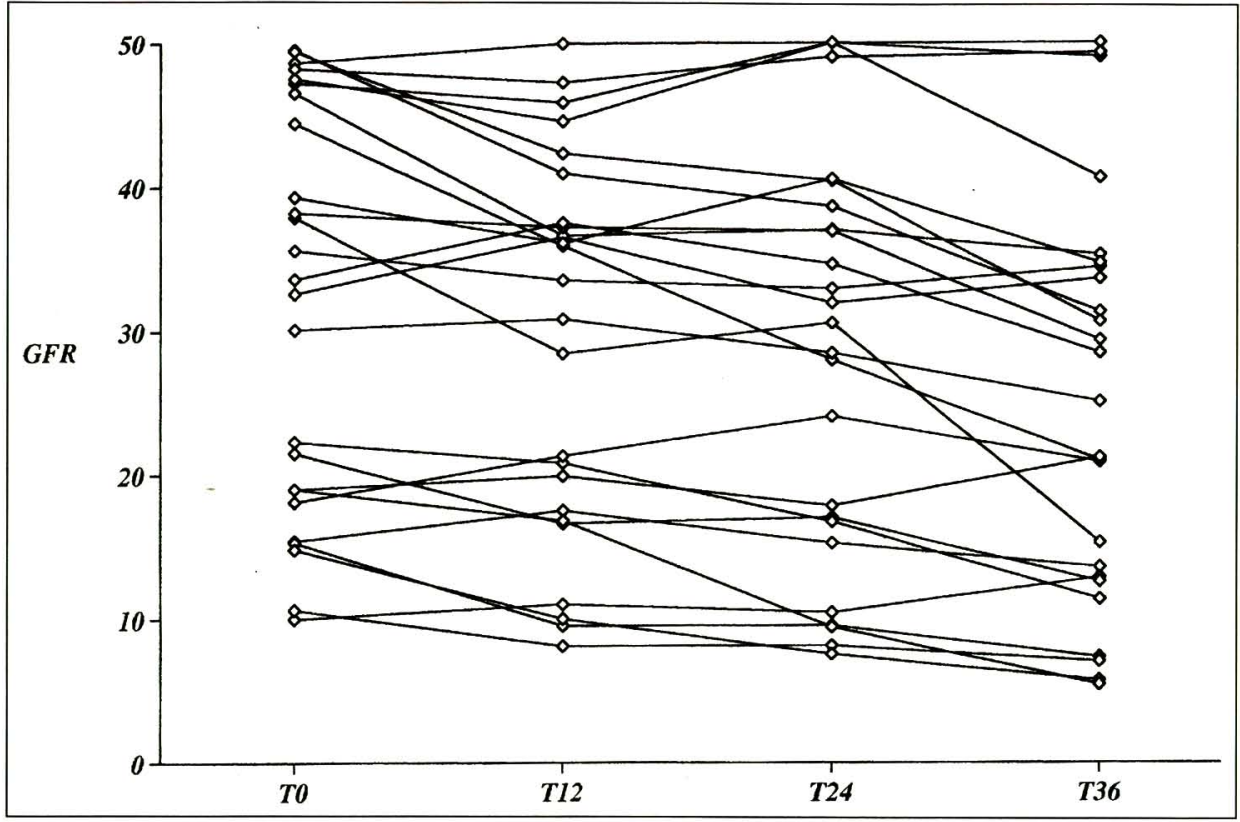

Fig. 1 - Andamento della funzione renale espresso come clearance della creatinina (GFR) in 25 bambini affetti da IRC.

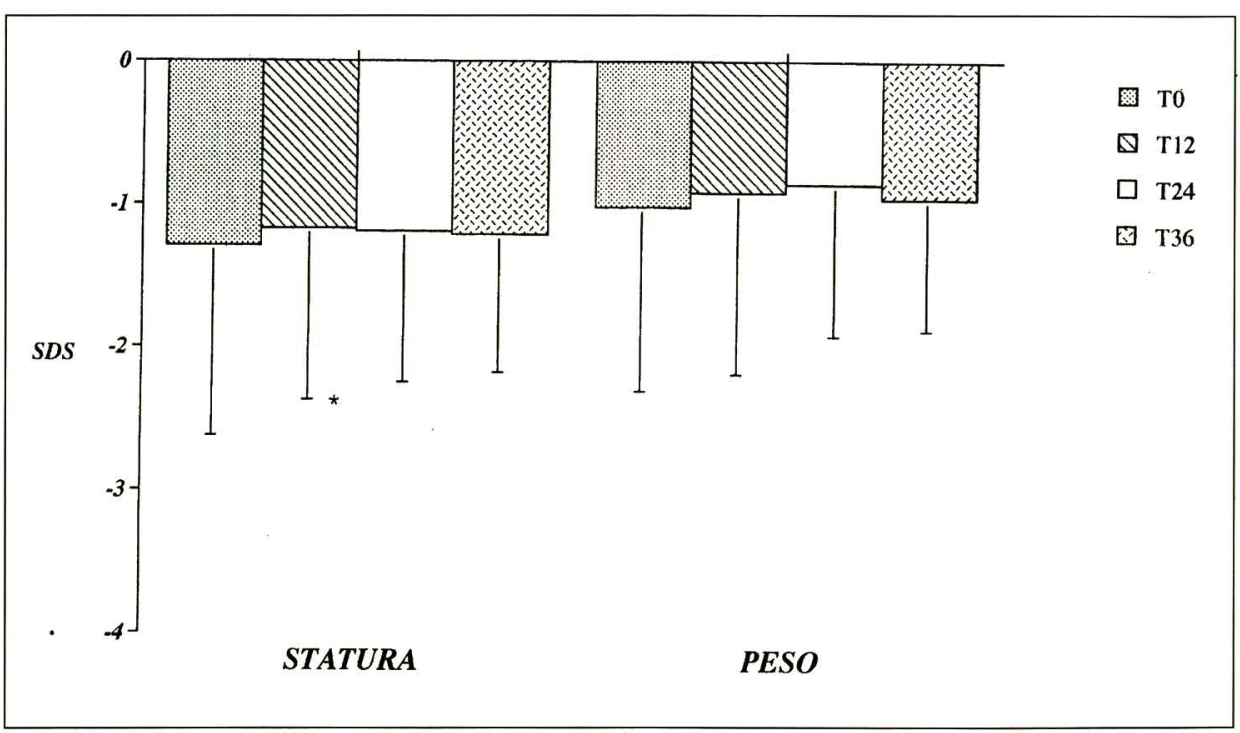

Fig. 2 - Statura e peso espressi in SDS in 25 bambini affetti da IRC (*T12 vs T0, p<0.05).

Nella Tabella I sono riportati i valori degli altri parametri antropometrici studiati. Il BMI si è mantenuto su valori stabili mostrando un significativo miglioramento al $36^{\circ}$ mese di osservazione. Il dato antropometrico più importante è rappresentato dall'aumento significativo dei parametri indicatori della massa muscolare (MAMC, AMA). Nella Figura 3 sono presentati i parametri derivati dall'analisi bioimpedenziometrica. Tutti i parametri analizzati si sono mantenuti costanti nel tempo senza evidenziare differenze significative ad esclusione della FFM (T12 vs T0 $\mathrm{p}<0.05)$. Nella Tabella II sono riportati i 
TAB. I - PARAMETRI ANTROPOMETRICI IN 25 BAMBINI AFFETTI DA IRC

\begin{tabular}{lcccc}
\hline & T0 & T12 & T24 & T36 \\
\hline BMI & $16.68 \pm 2.86$ & $16.88 \pm 3.03$ & $17.35 \pm 3.15$ & $17.46 \pm 2.68^{*}$ \\
TSF $(\mathrm{mm})$ & $8.35 \pm 4.39$ & $7.92 \pm 4.21$ & $8.88 \pm 4.13$ & $8.27 \pm 3.44$ \\
SSF $(\mathrm{mm})$ & $6.78 \pm 3.88$ & $7.29 \pm 4.47^{*}$ & $7.47 \pm 4.03^{*}$ & $7.00 \pm 2.23$ \\
MAC $(\mathrm{mm})$ & $181.2 \pm 35.7$ & $189.8 \pm 37.8^{*}$ & $199.7 \pm 36.2^{* *}$ & $202.7 \pm 35.7^{* *}$ \\
MAMC $(\mathrm{mm})$ & $155.2 \pm 28.2$ & $161.9 \pm 32.5$ & $172.0 \pm 31.4^{* *}$ & $176.5 \pm 34.3^{* *}$ \\
AMA $\left(\mathrm{mm}^{2}\right)$ & $1977.4 \pm 731.3$ & $2168.9 \pm 925.4$ & $2431.5 \pm 899.4^{* *}$ & $2574.2 \pm 997.7^{* *}$ \\
AFA $\left(\mathrm{mm}^{2}\right)$ & $733.9 \pm 538.4$ & $768.3 \pm 531.4$ & $793.7 \pm 499.8$ & $790.9 \pm 387.9$ \\
\hline
\end{tabular}

$(\mathrm{BMI}=$ body mass index, $\mathrm{TSF}=$ plica tricipitale, $\mathrm{SSF}=$ plica sottoscapolare, $\mathrm{MAC}=$ circonferenza del braccio, $\mathrm{MAMC}=$ circonferenza muscolare del braccio, $\mathrm{AMA}=$ area trasversale del muscolo dell'arto superiore, $\mathrm{AFA}=$ area trasversale del grasso dell'arto superiore)

La significatività si riferisce al tempo indicato dall'asterisco vs T0 $\left({ }^{*} \mathrm{p}<0.05, * * \mathrm{p}<0.001\right)$

TAB. II - PARAMETRI EMATOCHIMICI RILEVATI IN 25 BAMBINI AFFETTI DA IRC

\begin{tabular}{|c|c|c|c|c|}
\hline & T0 & $\mathrm{T} 12$ & $\mathrm{~T} 24$ & T36 \\
\hline $\begin{array}{l}\mathrm{HCO}_{3} \\
(\mathrm{mEq} / \mathrm{L})\end{array}$ & $21.46 \pm 1.64$ & $22.03 \pm 2.03$ & $22.79 \pm 2.76^{*}$ & $24.09 \pm 2.59^{* *}$ \\
\hline $\begin{array}{l}\text { Colesterolo } \\
(\mathrm{mg} / \mathrm{dl})\end{array}$ & $187.9 \pm 33.2$ & $191.1 \pm 23.1$ & $175.9 \pm 35.0$ & $173.5 \pm 33.2$ \\
\hline $\begin{array}{l}\text { Trigliceridi } \\
(\mathrm{mg} / \mathrm{dl})\end{array}$ & $126.8 \pm 44.3$ & $121.3 \pm 49.9$ & $111.6 \pm 40.6$ & $98.2 \pm 31.9$ \\
\hline $\begin{array}{l}\text { Proteine totali } \\
(\mathrm{g} / \mathrm{dl})\end{array}$ & $7.50 \pm 0.36$ & $7.47 \pm 0.53$ & $7.60 \pm 0.5$ & $7.46 \pm 0.32$ \\
\hline $\begin{array}{l}\text { Albumina } \\
(\mathrm{g} / \mathrm{dl})\end{array}$ & $4.80 \pm 0.32$ & $4.85 \pm 0.31$ & $4.90 \pm 0.41$ & $4.60 \pm 0.34$ \\
\hline
\end{tabular}

(* T24 vs T0, $<<0.05 ; * *$ T36 vs T0, $\mathrm{p}<0.001$ )

dati biochimici rilevati ai vari tempi di osservazione. L'equilibrio acido-base è risultato ben controllato per tutto il periodo di osservazione evidenziando un miglioramento significativo nel tempo. Un buon risultato si è osservato nel controllo dei lipidi ematici con un graduale decremento dei livelli di colesterolo e dei trigliceridi. I livelli ematici di proteine e albumina si sono mantenuti costanti nel tempo.

\section{Discussione}

La valutazione dello stato nutrizionale del bambino affetto da IRC rappresenta uno dei principali campi di intervento clinico del nefrologo pediatra. L'interesse verso questo tipo di studio è aumentato notevolmente negli ultimi anni. La dieta ha un ruolo fondamentale nel mantenimento di un adeguato stato nutrizio- nale e mentre un tempo era considerata un supporto utile a diminuire $\mathrm{i}$ sintomi correlati alla sindrome uremica, negli ultimi anni ha cercato di proporsi come mezzo per rallentare l'evoluzione dell'insufficienza renale $(3,33,35,38,39$, 42); nessuno studio nel bambino, però, ha finora dato prova di questa possibilità. I principi sui quali si basa il trattamento dietetico nel bambino sono in gran parte sovrapponibili a quelli dell'adulto e cioè 


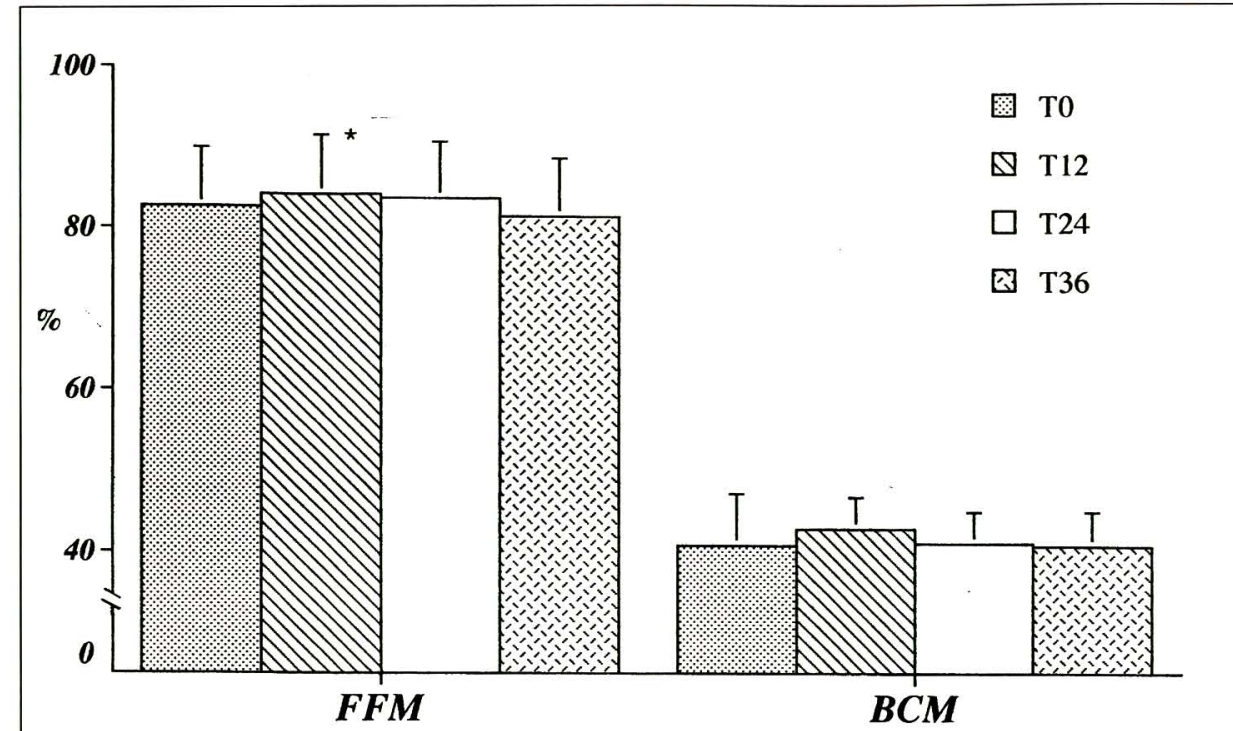

$\mathrm{FFM}=$ massa magra, $\mathrm{BCM}=$ massa cellulare, $\mathrm{TBW}=$ acqua corporea totale, $\mathrm{ECW}=\mathrm{ac}-$ qua extracellulare.

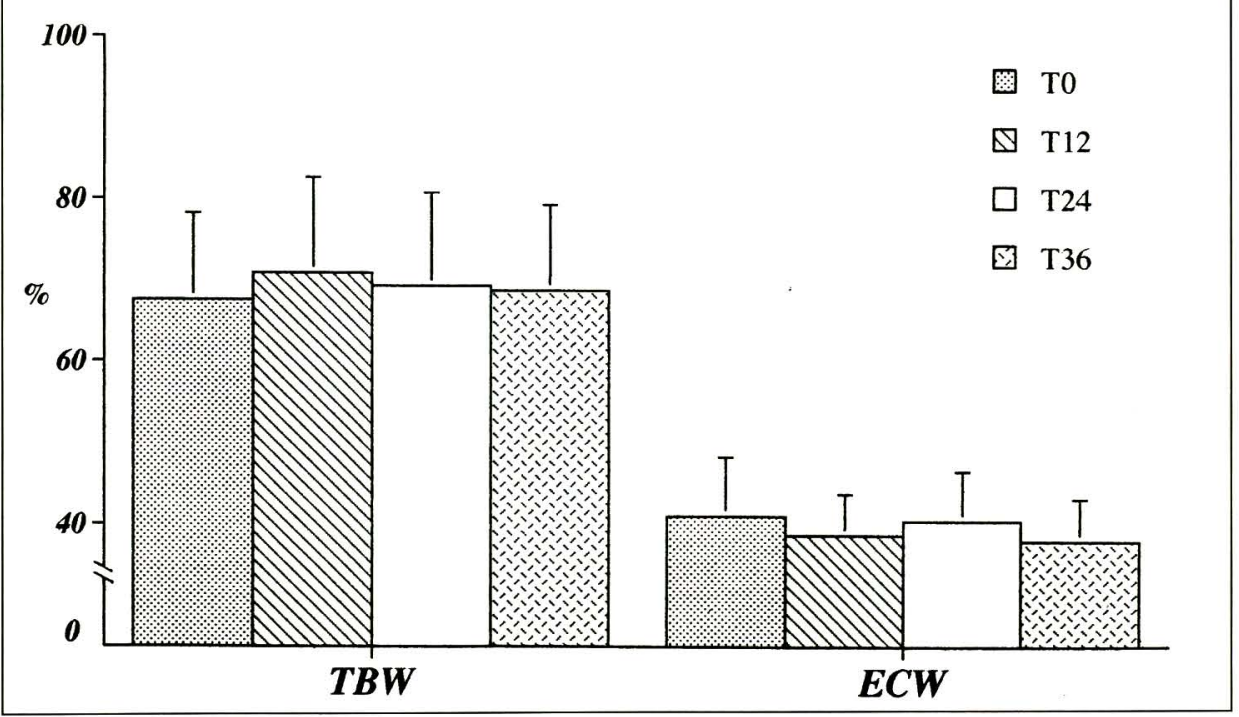

Fig. 3 - Parametri derivati dall' analisi bioimpedenziometrica espressi in valori percentuali in 25 bambini affetti da IRC $(*$ T12 vs T0, p<0.05).

devono cercare di mantenere gli equilibri acido-base e idroelettrolitico, diminuire l'accumulo di cataboliti azotati, prevenire o modificare alcune complicanze; in più devono cercare di mantenere un accrescimento staturo-ponderale il più possibile vicino alla norma.

Quando si prescrive una dieta ipoproteica nel paziente pediatrico occorre considerare $\mathrm{i}$ potenziali effetti negativi che l'eventuale malnutrizione proteica potrebbe avere sulla crescita. E opportuno nella prescrizione di una dieta ipoproteica che il $50 \%$ delle proteine fornite sia di elevato valore biologico e l'apporto calorico sia mantenuto il più vicino possibile al $100 \%$ delle calorie raccomandate per l'età $(7,24,27 ; 48,49)$. Importante è inoltre, quando si prescrive una dieta ipoproteica, valutarne gli effetti sullo stato nutrizionale. I parametri antropometrici valutati nei nostri pazienti sono da tempo utilizzati e validati da vari Autori. Il BMI è un parametro da molti con- siderato un ottimo indicatore dello stato nutrizionale in quanto indipendente dall'età, questo indice risulta generalmente diminuito nei pazienti con IRC avanzata a seguito di una riduzione della massa muscolare e del tessuto adiposo (1). Infatti il ritmo di produzione di creatinina, usato come indice di massa muscolare, è ridotto nell'uremia grave, così come lo spessore delle pliche cutanee $(5,18)$. Nei nostri pazienti il BMI si è mantenuto costante evidenziando un lieve miglioramento nel tempo. Per quanto riguarda la circonferenza del braccio anch'essa, di per sé, non fornisce un dato certo di malnutrizione ma, se viene associata alla plica tricipitale, può essere ricavata la circonferenza muscolare del braccio (MAMC), parametro indicatore della massa muscolare e quindi della principale riserva di proteine $(22,25,31)$. Dai dati antropometrici basali possono essere derivati parametri più precisi quali le aree trasversali del muscolo e del grasso dell'arto superiore (AMA, AFA) (17). Nei nostri pazienti abbiamo osservato un significativo aumento dei parametri indicatori della massa muscolare, MAMC e AMA, durante tutto il periodo di osservazione, mentre non si avevano differenze significative degli indicatori delle riserve di grasso. I primi studi riguardanti la bioimpedenziometria furono compiuti da Thomasset agli inizi degli anni '60 quando aveva evidenziato le relazioni esistenti tra misure dell'impedenza elettrica dell'organismo e quantità di acqua corporea totale (46). Da allora numerosi studi impedenziometrici sono stati compiuti nell'adulto da vari Autori $(2,4,6$, $9,10,11,13,14,19,20,23,34,36,45)$ che hanno confermato la validità di questa tecnica confrontandone i risultati con quelli ottenuti con altre metodiche già note quali la densitometria e le diluizioni isotopiche. L'utilizzo della bioimpedenziometria si è sviluppato anche in campo pediatrico $(16,21,28,29,30,40,43)$. Bisogna peraltro ricordare che le varie tecniche usate per risalire alla composizione corporea, convalidate in bambini normali, possono non essere del tutto attendibili quando vengono applicate a soggetti malnutriti. Questi pazienti presentano infatti una densità corporea alterata, disfunzioni cellulari con anomala distribuzione intra- ed extracellulare di marcatori utilizzati per effettuare alcune misurazioni, patologiche compartimentalizzazioni dell'acqua, perdite di peso non 
uniformi in tutti gli organi, modificazioni nella composizione del tessuto adiposo con aumento della sua composizione in acqua e collageno (12). Sebbene esistano questi limiti l'utilizzo della bioimpedenziometria presenta numerosi vantaggi quali la non invasività, il basso costo e la ripetibilità. Tutto ciò permette di seguire in modo più preciso i pazienti pediatrici con IRC e valutare gli effetti del trattamento dietetico $(4,35)$. Attraverso l'impedenziometria sono state descritte nei soggetti con IRC diminuzioni della massa magra (FFM) e aumento dell'acqua corporea totale (TBW) $(12,15$, 37). I dati impedenziometrici rilevati nei nostri pazienti hanno evidenziato un buon mantenimento della FFM con valori medi superiori all' $80 \%$ e una normale distribuzione dell'acqua corporea. L'esame dei dati ottenuti in questo gruppo di pazienti, che si caratterizza per la completezza dei dati antropometrici e la associazione dei dati rilevati con la bioimpedenziometria, e per il fatto di essere stato seguito per un periodo lungo di tempo (36 mesi) permette di trarre alcune considerazioni.

Analizzando tutti i dati ottenuti con le diverse tecniche utilizzate possiamo affermare che è possibile mantenere uno stato nutrizionale adeguato pur in presenza di una compromissione della funzione renale e di una progressione del danno stesso. Tale risultato è riconducibile ad una terapia dietetica e medica appropriate ma anche all'uso di queste tecniche che, a completamento delle valutazioni più tradizionali, permettono di programmare variazioni terapeutiche e dietetiche e di verificarne l'utilità.

\section{Riassunto}

Scopo del nostro lavoro è stato quello di analizzare lo stato nutrizionale di 25 bambini affetti da insuffilcienza renale cronica (IRC) seguiti longitudinalmente per un periodo di 36 mesi. All'inizio dell'osservazione i pazienti presentavano un'età media pari a $8.81 \pm 3.74$ e un filtrato glomerulare pari a $32.48 \pm 13.68$ $\mathrm{ml} / \mathrm{min} / \mathrm{m}^{2} 1.73$. Sono stati valutati i seguenti parametri antropometrici: statura, peso, body mass index (BMI), plica tricipitale, plica sottoscapolare, circonferenza del braccio, circonferenza muscolare del braccio, area trasversale del muscolo e area trasversale del grasso dell'arto superiore. Per mezzo di un analizzatore BLA 101 sono state misurate resistenza e reattanza e sono state calcolate acqua corporea totale, acqua extracellulare, massa magra, massa cellulare. Sono stati inoltre rilevati bicarbonatemia, colesterolemia, trigliceridemia, proteinemia e albuminemia. Ogni sei mesi venivano rilevati inoltre gli apporti alimentari mediante registrazione dietetica. Nei nostri pazienti peso e statura si sono mantenuti costanti senza evidenziare differenze significative tra i diversi periodi di osservazione ad eccezione della statura rilevata al tempo 12. Il dato antropometrico più importante è rappresentato dall'aumento significativo dei parametri indicativi della massa muscolare. L'analisi impedenziometrica ha evidenziato un buon mantenimento della massa magra e una normale distribuzione dell'acqua corporea. Facendo un esame globale dei dati ottenuti con le diverse tecniche utilizzate possiamo affermare che è possibile mantenere uno stato nutrizionale adeguato anche in presenza di una compromissione della funzione renale.

\section{BIBLIOGRAFIA}

1.

Abitbol C, Warady BA, Massie $\mathrm{MD}$, et al. Linear growth and anthropometric and nutritional measurements in children with mild to moderate renal insufficiency: a report of the growth failure in children with renal diseases study. J Pediatr 1990; 116: S46S54.

2. Anonymus. Bioelectrical impedance and body composition. Lancet 1992; 340, N 8834-5: 1511.

3. Barsotti G, Morelli E, Giannoni A, Guiducci A, Lupetti S, Giovannetti S. Restricted phosphorus and nitrogen intake to slow the progression of chronic renal failure: a controlled trial. Kidney Int 1983; 24 (Suppl 16): S278-84.

4. Ballmgarter RN, Chumlea WC, Roche AF. Bioelectrical impedance phase angle and body composition. Am J Clin Nutr 1988; 48: 16-23.

5. Boileau RB, Horstman DH, Buskirk ER, Mendez J. The usefulness of urinary creatinine excretion in estimating body composition. Med Sci Sports 1972; 4: 85-90.

6. Cameron Chumlea C, Baumgartner RN, Roche AR. Specific resistivity used to estimate fat-free mass from segmental body measures of bioelectric impedance. Am J Clin Nutr 1988; 48: 7-15.

7. Chantler C. Nutritional assessment and management of children with renal insufficiency. In: Fine RN, Gruskin AB eds. Endstage Renal Disease in Children. Philadelphia B Sauders, 1984; 193.

8. Chantler C, Holliday MA. Growth in children ith renal disea- 
se with particular reference to the effects of calorie malnutrition: a review. Clin Nephrol 1973; 1: 230.

9. Chumlea WC, Baumgartner RN, Mitchell CO. The use of segmental bioelectric impedance in estimating body composition. Basic Life Sci 1990; 55: 375-85.

10. Chumlea WC, Baumgartner RN. Bioelectric impedance methods for the estimation of body composition. Can J Sport Sci 1990; 15(3): 172-9.

11. Chumlea WC, Guo SS. Bioelectrical impedance and body composition: present status and future directions. Nutr Rev 1994; 52 (4): 123-31.

12. Cohn SH. How valid are bioelectric impedance measurements in body composition studies. Am J Clin Nutr 1985; 42: 889-90.

13. Cunningham JJ. New approaches to the noninvasive assessment of body composition: bioelectrical impedance analysis and total body electrical conductivity. Nutr Int 1987; 3: 1.

14. Deurenberg P, Weststrate JA, Hautvast JGAJ. Changes in fat-free mass during eight loss measured by bioelectrical impedance and by densitometry. Am J Nutr 1989; 49: 33-6.

15. Diaz EO, Villar J, Immink M, Gonzales T. Bioimpedance or anthropometry? Eur J Clin Nutr 1989; 43: 129-37.

16. Ferrante E, Pitzalis G, Deganello F, Galastri E, Sciarpelletti R, Imperato $\mathrm{C}$. The evaluation of body composition in children by anthropometry and impedance measurement. Minerva Pediatr 1993; 45 (7-8): 289-98.

17. Frisancho AR. New norms of upper limb fat and muscle areas for assessment of nutritional status. Am J Clin Nutr 1981; 34: 2540-5.
18. Forbes GB, Bruining GJ. Urinary creatinine excretion and lean body mass. Med Sci Sports 1972; 4: 85-90.

19. Forbes GB. Bioelectrical impedance and body composition: present status and future direction. Nutrition Reviews 1994; 52, N 9: 323.

20. Gray DS. Changes in bioelectrical impedance during fasting. Am J Clin Nutr 1988; 47: 7-14.

21. Guo S, Roche AF, Houtkooper L. Fat-free mass in children and young adults predicted from bioelectric impedance and anthropometric variables. Am J Clin Nutr 1989; 50: 43543.

22. Gurney JM, Jeliffe DB. Arm anthropometry in nutritional assessment: nomogram for rapid calculation of muscle circumference and cross-sectional muscle over fat areas. Am J Clin Nutr 1973; 26: 912-5.

23. Heitmann BL. Impedance: a valid method in assessment of body composition? Eur J Clin Nutr 1994; 48 (4): 228-40.

24. Hellerstein S, Holliday MA, Grupe W et al. Nutritional menagement of children with chronic renal failure. Pediatr Nephrol 1987; 1: 195-211.

25. Heymsfield SB, McMannus C, Smith J, Stevens V, Nixon DW. Anthropometric measurement of muscle mass: revised equations for calculating bone-free arm muscle area. Am J Clin Nutr 1982; 36: $680-90$.

26. Holliday MA. Nutritional aspects of renal diseases in children and adults. Hosp Pract 1983; 18: 179-93.

27. Holliday MA. Protein intake, renal function and growth in chronic renal failure. In: Mitch WE ed. The progressive nature of renal disease. New York: Churchill Livingstone, 1986; 245-62.
28. Houtkooper LB, Lohman TG, Going SB, Hall MC. Validity of bioelectric impedance for body composition assessment in children. J Appl Physiol 1989; 66 (2): 814-21.

29. Houtkooper LB, Going SB, Lohman TG, Roche AF, VanLoan M. Bioelectrical impedance estimation of fat-free body mass in children and youth: a cross-validation study. J Appl Phisiol 1992; 72 (1): 366-73.

30. Iwata K, Satou Y, Iwata F et al. Assessment of body composition measured by bioelectrical impedance in children. Acta Paediatr Jpn 1993; 35 (5): 369-72.

31. Jeliffe EFP, Jeliffe DB. The arm circumference as a public health index of protein-calorie malnutrition of early chilhood. J Trop Pediatr 1969, 15: 179-92.

32. Jones RWA, Ridgen SP, Barratt TM et al. The effect of chronic renal failure in infancy on growth nutritional status and body composition. Pediatr Res 1982; 16: 78491.

33. Klahr S, Buerkert J, Purkerson ML. Role of dietaly factors in the progression of chronic renal disease. Kidney Int 1983; 24: 57987.

34. Kushner RF. Bioelectrical impedance analysis: a review of principles and applications. J Am Coll Nutr 1992; 11 (2): 199-209.

35. Locatelli F, Alberti D, Graziani G, Buccianti G, Redaelli B, Giangrande $A$, and the Northen Italian Cooperative Study Group. Prospective, randomized, multicentre trial of the effect of protein restriction on progression of chronic renal insufficiency. Lancet 1991; 337: 1299.

36. Lukaski HC, Johnson PE, Bolonchuc W, Likken GI. Assessment of fat-free mass using bioelectrical impedance measurement of the human body. Am J Clin Nutr 1985; 42: 810-7. 
37. Mackie A, Hannan J, Tothill P. An introduction to body composition models used in nutritional studies. Clin Phys Physio Meas 1989; 10, 4: 297-310.

38. Maschio G, Oldrizzi L, Tessitore N, et al. Effect of dietary protein and phosphorus restriction on the progression of early renal failure. Kidney Int 1982; 22: 371-6.

39. Mitch WE. The influence of the diet on the progression of renal insufficiency. Ann Rev Med 1984; 35: 249-64.

40. Mayfield S, Uauy R, Waidelich D. Body composition of low-birth-weight infants determined by using bioelectrical resistance and reactance. Am J Clin Nutr 1991; 54: 296-303.

41. Perfumo F. Insufficienza renale cronica. In: Gusmano R, Perfumo $\mathrm{F}$ eds. Le malattie renali nel bambino. Milano: Wichtig, 1991: 395-406.

42. Rosman JB, Meijer S, Sluiter WJ, Ter Wee PM, Piers-Brecht TP, Donker AJM. Prospective randomised trial of early dietary protein restriction in chronic renal failure. Lancet 1984; 2: 1293-5.

43. Sacco P, Perfumo F, Delucchi P, Gusmano R. Valutazione dello stato nutrizionale nel bambino affetto da insufficienza renale cronica: studio antropometrico e bioimpedenziometrico. Gaslini, 1994; 26: 215-21.

44. Salusky IB, Fine RN, Nelson $P$ et al. Nutritional status of pediatric patients undergoing CAPD. Kidney Int 1982; 21: 177-81.

45. Segal KR, Loan MV, Fitzgerald $\mathrm{P}$, Hodgdon JA, Van Itallie TB. Lean body mass estimation by bioelectrical impedance analysis: four site cross-validation study. Am J Clin Nutr 1988; 47: 7-14.

46. Thomasset A. Bio-electrical properties of tissue impedance measurements. Lyon Med 1962; 207: 1017-8.
47. Wassner WJ. The role of nutrition in the care of children with renal insufficiency. Pediatr Clin N Am 1982; 29: 973-90.

48. Wassner WJ, Abitbol C, Alexander S. Nutrition requirements for infants with renal failure. Am J Kidney Dis 1986; 7 : 300-5.

49. Wingen AM, Fabian-Bach C, Mehls O. Evaluation of protein intake by dietary diares and urea-N excretion in children with chronic renal failure. Clin Nephrol 1993; 40 (4): 208-15. 